ORIGINAL ARTICLE

\title{
Agreement between an arterial blood gas analyser and a venous blood analyser in the measurement of potassium in patients in cardiac arrest
}

\author{
H L M Johnston, R Murphy
}

Emerg Med J 2005;22:269-271. doi: 10.1136/emj.2003.013599

Background and objective: Rapid measurement of potassium is crucial in the resuscitation of patients in cardiac arrest. This is often done on an arterial blood gas sample taken during resuscitation and analysed in an emergency department based blood gas analyser. No-one has assessed how accurate or reliable this is when compared with the traditional method of sending a venous sample to the laboratory for standard analysis. This study looked at the agreement between potassium measurements in arterial blood gas samples and venous blood samples in patients in cardiac arrest.

See end of article for authors' affiliations ....................

Correspondence to: R Murphy, rossmurphy@ doctors.org.uk

Accepted for publication 1 April 2004
Method: Arterial and venous blood samples were taken at the same time and analysed in the usual way from 50 patients in cardiac arrest. It was found that the mean difference between each pair of arterial and venous potassium measurements was low at $0.106 \mathrm{mmol} / \mathrm{l}$. However, the standard deviation of these differences and subsequently the $95 \%$ limits of agreement were wide $(-1.182 \mathrm{mmol} / \mathrm{l}$ to $1.394 \mathrm{mmol} / \mathrm{l})-$ that is, $95 \%$ of differences will lie between these limits. It is felt that these limits are too wide for safe use in clinical practice.

Conclusion: Based on these results, it is advised that arterial blood gas analysers should be used with caution to measure potassium in patients in cardiac arrest.
A bnormalities of potassium concentration are considered reversible causes of cardiac arrest. ${ }^{1}$ Hyperkalaemia causes hyperpolarisation of the heart, bradycardia, and asystole. Hypokalaemia, especially in the setting of heart disease and digoxin toxicity, causes fatal cardiac arrhythmias. In addition, the potassium concentration in blood frequently changes during the course of a resuscitation. Just after cardiac arrest the concentration rises due to the release of potassium from underperfused cells. Then, as resuscitation gets underway, there is often a rebound fall as the circulation is restored and endogenous adrenaline and exogenous drugs take effect. $^{2}$

It is, therefore, important to measure potassium as soon as possible during the course of a resuscitation. This has traditionally been done by taking a venous sample and sending it to the biochemistry laboratory for standard analysis. This, however, can take some time. Many people now prefer to measure potassium on an arterial blood gas sample in a resuscitation room based blood gas analyser. This gives a much faster result, but it is not known how accurate this is when compared with a venous measurement.

In this study we aimed to determine the agreement between potassium measurements in venous and arterial blood gas samples in patients in cardiac arrest.

\section{MATERIALS AND METHODS}

The emergency department records of patients who presented in cardiac arrest over a 10 month period in 2001 were traced and examined. A sample size of 50 was considered appropriate as this is the minimum sample size recommended for a method comparison study. ${ }^{3}$ Patients were selected on the basis of both their arterial blood gas and venous laboratory blood results being available. This involved searching through emergency department, medical, and laboratory records until such records for 50 patients were obtained.

At our hospital during resuscitation the arterial and venous blood samples are collected from either the radial or femoral artery and a vein. The arterial sample is taken directly into a preheparinised syringe and placed immediately in the arterial blood gas analyser in the resuscitation room (ILl640 Blood Gas Analyser). It takes approximately two minutes for the results to be ready. The venous sample is collected and sent to a central laboratory in the hospital, via a tube transport system, for analysis (Olympus Analyser). It takes approximately 40 minutes for the results to be uploaded onto the hospital computer system or they can be obtained slightly more quickly via telephone.

For the present study both sets of results were checked to make sure the dates and times of collection matched for each patient. For venous samples the time recorded was the time at which the blood samples were received by the laboratory as an emergency sample. For arterial samples the time taken was the time at which the samples were analysed by the arterial blood gas analyser. These times were checked to ensure that there was never more than 10 minutes between them and the time at which the patient was recorded as entering the resuscitation room. These three times were recorded automatically by the laboratory and by the arterial blood gas analyser, and in the nursing notes, respectively.

The measurements for each patient were entered in a database for statistical analysis using Microsoft Excel for Windows (version 4.0, 1985-1997 Microsoft Corp, USA) and compared using methods described by Altman for assessing the agreement between two methods of clinical measurement. $^{3}$

\section{RESULTS}

Both potassium measurements for each patient are given in table 1 .

The arterial potassium measurements ranged between $2.7 \mathrm{mmol} / \mathrm{l}$ and $6.7 \mathrm{mmol} / \mathrm{l}$ (mean $4.17 \mathrm{mmol} / \mathrm{l}$ ). The venous measurements ranged between $2.5 \mathrm{mmol} / \mathrm{l}$ and $6.3 \mathrm{mmol} / \mathrm{l}$ (mean $4.06 \mathrm{mmol} / \mathrm{l}$ ). Pearson's correlation coefficient between the venous and arterial measurements was 0.73 . 
Table 1 Arterial and venous potassium levels of 50 patients in cardiac arrest.

\begin{tabular}{|c|c|c|}
\hline Patient & Arterial potassium ( $\mathrm{mmol} / \mathrm{l})$ & Venous potassium $(\mathrm{mmol} / \mathrm{l})$ \\
\hline 1 & 6.7 & 3.3 \\
\hline 2 & 2.7 & 2.5 \\
\hline 3 & 4.3 & 4.7 \\
\hline 4 & 4.6 & 4.4 \\
\hline 5 & 3.3 & 3.6 \\
\hline 6 & 5 & 4.2 \\
\hline 7 & 5 & 5.5 \\
\hline 8 & 4.1 & 4.6 \\
\hline 9 & 6 & 4.4 \\
\hline 10 & 5.1 & 5 \\
\hline 11 & 3.7 & 3.9 \\
\hline 12 & 5.9 & 5.5 \\
\hline 13 & 5.5 & 5.1 \\
\hline 14 & 4.2 & 4.9 \\
\hline 15 & 4.2 & 3.6 \\
\hline 16 & 3.3 & 3.7 \\
\hline 17 & 4.1 & 3.9 \\
\hline 18 & 5.8 & 5.1 \\
\hline 19 & 3.1 & 3.2 \\
\hline 20 & 3.3 & 3.6 \\
\hline 21 & 3.8 & 3.3 \\
\hline 22 & 5.2 & 5 \\
\hline 23 & 3.8 & 3.6 \\
\hline 24 & 5.4 & 5 \\
\hline 25 & 3.6 & 4.1 \\
\hline 26 & 4.3 & 4.1 \\
\hline 27 & 5.4 & 6.3 \\
\hline 28 & 3.4 & 3.5 \\
\hline 29 & 2.7 & 2.6 \\
\hline 30 & 3.4 & 3.2 \\
\hline 31 & 3.4 & 3.8 \\
\hline 32 & 3.4 & 3.4 \\
\hline 33 & 3.3 & 3.2 \\
\hline 34 & 4.4 & 4.1 \\
\hline 35 & 4.1 & 4.2 \\
\hline 36 & 4.3 & 4.9 \\
\hline 37 & 3.7 & 3.4 \\
\hline 38 & 4.9 & 5.3 \\
\hline 39 & 3.5 & 3.7 \\
\hline 40 & 3.7 & 3.7 \\
\hline 41 & 3.1 & 3.2 \\
\hline 42 & 3.1 & 2.8 \\
\hline 43 & 4.2 & 4.5 \\
\hline 44 & 4.7 & 4.2 \\
\hline 45 & 4.7 & 4.9 \\
\hline 46 & 3.4 & 3.6 \\
\hline 47 & 4 & 3.8 \\
\hline 48 & 4.1 & 4 \\
\hline 49 & 3.9 & 3.6 \\
\hline 50 & 3.7 & 3.5 \\
\hline
\end{tabular}

These measurements were plotted against each other with the line of equality (fig 1). The line of equality is the line along which all points would lie if there was exact agreement.

We analysed the results further to better assess the agreement. The differences between each pair of arterial and venous measurements ranged between $-0.9 \mathrm{mmol} / \mathrm{l}$ and $3.4 \mathrm{mmol} / \mathrm{l}$ (mean (SD) $0.106 \mathrm{mmol} / \mathrm{l}(0.644 \mathrm{mmol} / \mathrm{l})$ ). Two standard deviations above and below this mean are the $95 \%$ limits of agreement; 95\% of differences will lie within these limits. We calculated these limits as $-1.182 \mathrm{mmol} / \mathrm{l}$ and $1.394 \mathrm{mmol} / \mathrm{l}$ (see fig 2 which plots the mean between each arterial and venous potassium measurement against the difference).

\section{DISCUSSION}

The speed with which test results become available has received a lot of attention recently with the development of near patient tests for a variety of clinical conditions. It is felt that because less time is spent waiting for results and because fewer staff are involved in processing each test this has benefits for patients and clinicians and that it is more cost

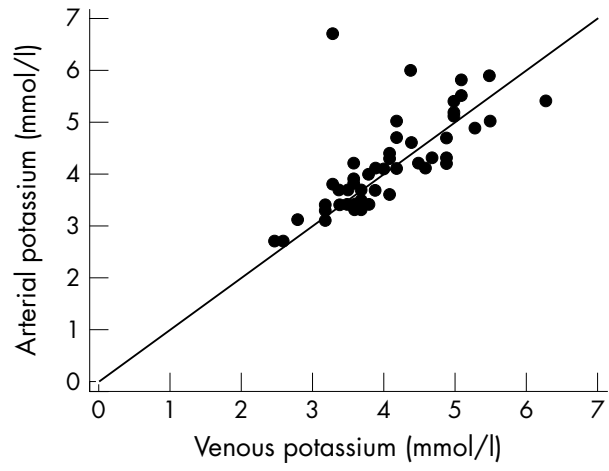

Figure 1 Arterial and venous potassium measurements with the line of equality.

effective, However, there is still some doubt about their accuracy and reliability with only $34 \%$ of clinicians in one study using them to guide patient care. ${ }^{4}$

Faster results are nowhere more important than in the emergency department where rule out and rule in strategies are needed to process different patient presentations. This is especially true in cardiac arrest when identifying treatable causes of the arrest is given a high priority. Arterial blood gas analysers are now regularly used to measure potassium in these situations. Our results show that the mean difference between each arterial and venous potassium measurement is low at $0.106 \mathrm{mmol} / \mathrm{l}$. However, the $95 \%$ limits of agreement were $-1.182 \mathrm{mmol} / \mathrm{l}$ to $+1.394 \mathrm{mmol} / \mathrm{l}$, which may be too wide for safe use in clinical practice. These results are consistent with a previous report which also identified wide limits of agreement although in a sample of intensive care patients. ${ }^{5}$

There are a number of possible reasons for this poor agreement. We used different analysers for the arterial and venous samples so a lack of agreement between the analysers themselves may account for some of the differences. The accuracy of all laboratory equipment, however, is now upheld by regular calibration and quality control procedures and the machines used in this study were maintained according to the manufacturers' instructions. As the heparinised arterial sample is plasma and the venous sample is serum this would also account for some of the differences. A previous study, albeit with a small number of patients, found that the mean difference between plasma and serum potassium measured on the same analyser was $0.5 \mathrm{mmol} / \mathrm{l}$ with a range of $0.1-$ $1.1 \mathrm{mmol} / \mathrm{l}$. The measurements were always higher in the venous sample. ${ }^{6}$

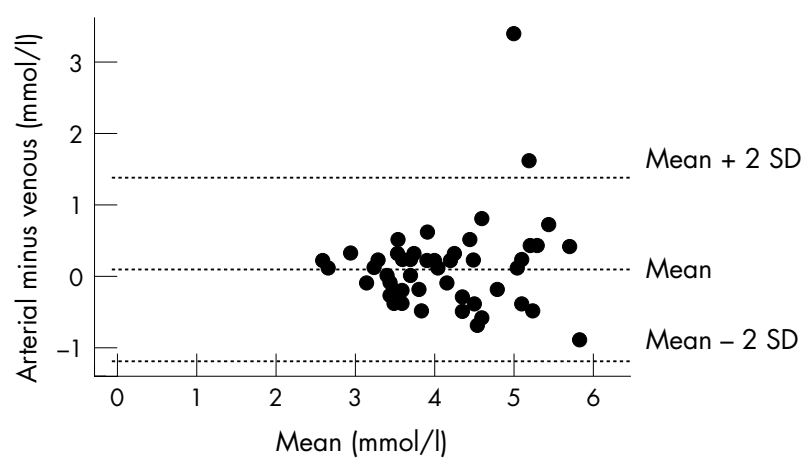

Figure 2 Difference against mean for each arterial and venous potassium measurement. The mean difference \pm 2 SD is shown by dotted lines. 
The two samples are also taken in different ways from different parts of an impaired blood circulation and this may give rise to further discrepancies. For the present study we felt as this was how the two measurements were made in real life it was important that this was how the results should be analysed.

The differences in this study, however, ranged between $-0.9 \mathrm{mmol} / \mathrm{l}$ and $3.4 \mathrm{mmol} / \mathrm{l}$. Some of these differences are too large to be explained by the above. We think that the main reason for such large differences is unrecognised haemolysis in the arterial sample. The venous samples sent to the laboratory are always centrifuged and then inspected visually for haemolysis. When haemolysis is detected the sample is discarded or no measurement is given for potassium. In our study these patients would therefore have been excluded. The arterial sample is not screened for haemolysis. It is put into the analyser and a potassium measurement is obtained regardless. The mean difference between our arterial and venous samples was positive indicating that potassium was usually measured higher in the arterial sample. In addition, whenever large differences occurred it was always the arterial potassium that was higher which supports our conclusions.

Our study did not aim to establish the effect of these differences on patient care and outcome and any risk at present remains theoretical. However, we feel that if a false high potassium result is obtained for a patient in cardiac arrest who has a low or normal potassium this could result in inappropriate or no treatment when action is urgently needed.
As a result of this study, we advise caution when using arterial blood gas analysers to measure potassium in patients in cardiac arrest. In future it may be useful to develop a means to identify haemolysis in arterial blood gas samples to eliminate potentially unreliable results.

\section{Authors' affiliations}

H L M Johnston, The Faculty of Medicine, University of Edinburgh, Edinburgh, UK

R Murphy, Accident and Emergency Department, Royal Infirmary of Edinburgh, Edinburgh, UK

Competing interests: none declared

\section{REFERENCES}

1 Resuscitation Council (UK). Advanced Life Support Course Provider Manual, 3rd edn. London: Resuscitation Council, 1998.

2 Salerno DM, Elsperger J, Helseth P, et al. Serum potassium, calcium, and magnesium after resuscitation from ventricular fibrillation: a canine study. J Am Coll Cardiol 1987; 10:178-85.

3 Altman DG. Some common problems in medical research. In: Altman DG, ed Practical statistics for medical research. London: Chapman and Hall/CRC, 1991:396-403.

4 Gray TA, Freedman DB, Burnett D, et al. Evidence based practice: clinicians' use and attitudes to near patient testing in hospitals. J Clin Pathol 1996;49:903-8.

5 King R, Campbell A. Performance of the Radiometer OSM3 and ABL505 blood gas analysers for determination of sodium, potassium and haemoglobin concentrations. Anaesthesia 2000;55:65-9.

6 Ward CF, Arkin DB, Benumof JL, et al. Arterial versus venous potassium: clinical implications. Crit Care Med 1976;6:335-6. 